\title{
Mathematical modelling of tumour acidity
}

\author{
Kieran Smallbone $^{\mathrm{a}, *}$, Robert A. Gatenby ${ }^{\mathrm{b}}$, Philip K. Maini ${ }^{\mathrm{c}, \mathrm{d}}$ \\ a Manchester Centre for Integrative Systems Biology, Manchester Interdisciplinary Biocentre, 131 Princess Street, Manchester M1 7DN, UK \\ ${ }^{\mathrm{b}}$ Moffitt Cancer Center, 12902 Magnolia Drive, Tampa, FL 33612, USA \\ ' Centre for Mathematical Biology, Mathematical Institute, 24-29 St Giles', Oxford OX1 3LB, UK \\ d Department of Biochemistry, Oxford Centre for Integrative Systems Biology, South Parks Road, Oxford OX13QU, UK
}

\section{A R T I C L E I N F O}

\section{Article history:}

Received 28 February 2008

Received in revised form

14 July 2008

Accepted 2 August 2008

Available online 7 August 2008

Keywords:

Tumour invasion

Glycolytic phenotype

Acidity

Mathematical modelling

\begin{abstract}
A B S T R A C T
Acid-mediated tumour invasion is receiving increasing experimental and clinical attention. Previous models proposed to describe this phenomenon failed to capture key properties of the system, such as the existence of the benign steady state, or predicted incorrectly the size of the inter-tissue gap. Here we show that taking proper account of quiescence ameliorates these drawbacks as well as revealing novel behaviour. The simplicity of the model allows us to fully identify the key parameters controlling different aspects of behaviour.
\end{abstract}

(c) 2008 Elsevier Ltd. All rights reserved.

\section{Introduction}

The tumour microenvironment is significantly different from that of normal tissue; its chaotic vasculature leads to a decrease in supply of essential nutrients and a decrease in the removal of waste products. One biomarker that has received much attention is tumour hypoxia (poor oxygenation). Near-zero oxygen levels are observed at distances of only $150 \mu \mathrm{m}$ from a feeding blood vessel (Thomlinson and Gray, 1955; Gatenby and Gillies, 2004). As such, areas of hypoxia are commonplace within tumours. Identifying the regions of hypoxia within tumours has been a focus of recent research, as cells residing within such regions are known to be resistant to various radio- and chemo-therapeutic strategies. Moreover, cells subjected to chronic hypoxia are found to be more aggressive, displaying increased metastasis, invasion and mutation (Gillies et al., 2002).

Acidity also plays a key role in tumour development. Like hypoxia, regions of low $\mathrm{pH}$ are commonplace within tumours. Moreover, the effects of acidosis are similar to those of hypoxia, with acidosis promoting metastasis, invasion and mutation (Gillies et al., 2002). However, unlike hypoxia, there has been relatively little research into acidity as a factor for promoting tumour development. The reasons for this are unclear; it may be that many investigators assume that the acidity is simply a byproduct of low oxygen levels. Cells respond to periods of

\footnotetext{
* Corresponding author. Tel.: +441613065146; fax: +441613065201.

E-mail address: kieran.smallbone@manchester.ac.uk (K. Smallbone).
}

hypoxia by converting to anaerobic respiration, or glycolysis, which in turn produces lactic acid and brings about lower tissue $\mathrm{pH}$. However, the work of Warburg early in the last century (Warburg, 1930) showed that the increased reliance on glycolysis to produce energy in many aggressive tumours occurs even in the presence of sufficient oxygen. As such, tumour acidification is an intrinsic property of both poor vasculature and altered tumour cell metabolism, and occurs independently of hypoxia.

In an earlier work (Smallbone et al., 2005), we developed a simple model of (spherically symmetric) tumour growth to examine the role of acidosis in the interaction between normal and tumour cell populations. Assuming that a mass of tumour cells displays typical characteristics of increased acid production coupled with a resistance to low $\mathrm{pH}$, we predicted that the hydrogen ions produced by the tumour will diffuse into the surrounding normal tissue, inducing cellular death. Thus acidity provides a simple mechanism for cancer invasion. Both vascular and avascular tumour dynamics were investigated, and a number of different potential behaviours arose. Whilst an avascular tumour always proceeds to a benign steady state, a vascular tumour may display either benign or invasive dynamics, depending on the value of a critical bifurcation parameter. The model also predicted the development of an experimentally observed acellular gap separating the advancing tumour and receding host tissue fronts (Gatenby and Gawlinski, 1996).

In this paper, we extend the previous model through the inclusion of quiescent (non-proliferating) tumour cells. Within avascular tumours, in particular, the vast majority of viable cells are quiescent, with active cells restricted to the nutrient-rich 
outer rim. These quiescent cells are essentially metabolically inactive, producing significantly less acid than their proliferating counterparts. By considering both active and quiescent cells, we give a more physiologically accurate description of the acidity in and around the tumour tissue. Moreover, the interfacial gap is predicted to be an order of magnitude smaller than the tumour radius at equilibrium, in line with experimental evidence.

\section{Model development}

We model the tumour as a sphere of radius $R_{M}$ and assume that spherical symmetry prevails at all times. Let $H$ denote the extracellular concentration of excess hydrogen ions, where excess means above its normal level of $10^{-7.25} \mathrm{M} \equiv \mathrm{pH} 7.25$. We assume that there is a sharp acid threshold concentration $H_{D}$ above which tumour cells cannot survive. Similarly, normal cells die when this concentration $H$ rises above $H_{N}$. Tumour cells are relatively resistant to extracellular acidity due to increased $\mathrm{Na}^{+} / \mathrm{H}^{+}$antiport activity and mutations in acid-induced apoptosis pathways, hence $H_{N} \ll H_{D}$. High levels of acidity can also induce tumour cells to cease proliferation, i.e. become quiescent (Casciari et al., 1992). Specifically, acidosis promotes the production of hypoxia inducible factor 1 (HIF-1); via a cyclin-dependent kinase inhibitor, p27, HIF-1 acts to inhibit the cell cycle (Goda et al., 2003; Murphy et al., 2004). Hence we assume that there also exists a sharp acidity threshold $H_{Q}$ above which tumour cells cease proliferation.

These assumptions typically lead to a tumour geometry as presented in Fig. 1. Necrotic tissue is located at the core of the tumour $R<R_{D}$. Within the viable region $R_{D}<R<R_{M}$, the active proliferating tumour cells are restricted to the outer rim $R_{Q}<R<R_{M}$, whilst the region $R_{D}<R<R_{Q}$ contains quiescent cells. Each of the radii are defined by the acid levels at their boundary $-H\left(R_{i}\right)=H_{i}$, for $i=D, Q, N$. We define the acid production rate $\phi_{Q}$ per cell for quiescent cells and $\phi_{A}$ for active cells, where $\phi_{Q} \ll \phi_{A}$ as quiescent cells are essentially metabolically inactive, producing significantly less acid than proliferating cells

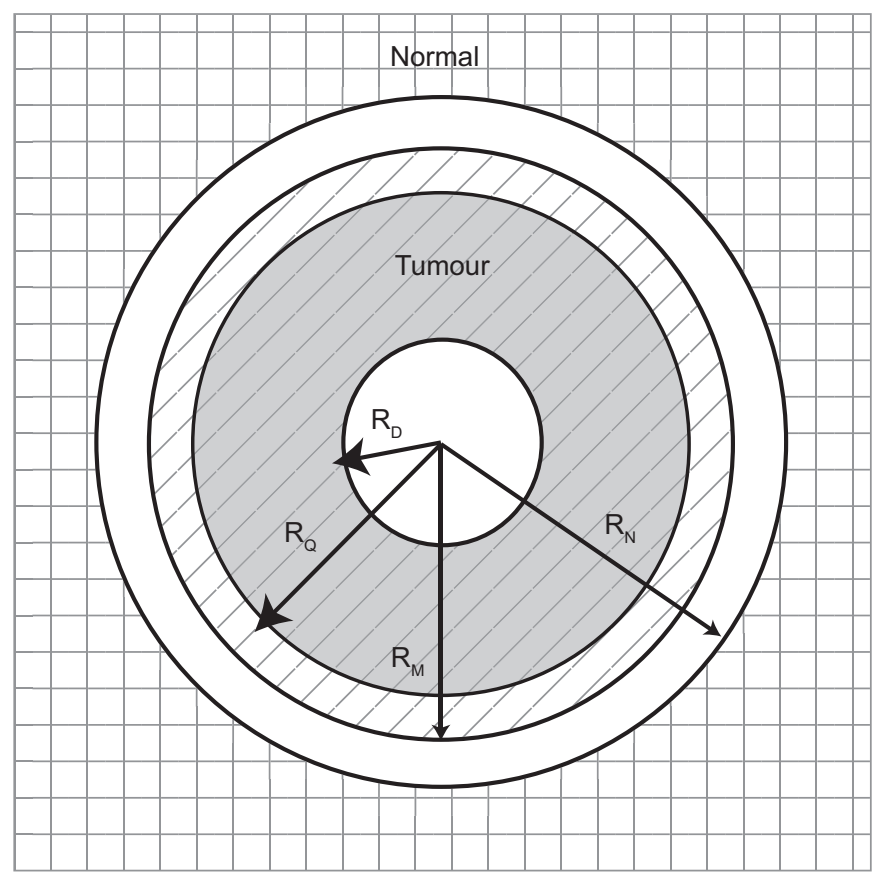

Fig. 1. Cross-section of a tumour and its surrounding tissue showing the central necrotic core, $R<R_{D}$, a layer of quiescent tumour cells $R_{D}<R<R_{Q}$, a layer of proliferating tumour cells $R_{Q}<R<R_{M}$, the acellular gap separating normal and tumour cell fronts $R_{M}<R<R_{N}$, and the normal cells $R_{N}<R$.
(Casciari et al., 1992). The primary mode for removal of acid from the system is through blood vessels and we assume that this occurs at a rate $r_{V}$ proportional to the local acid concentration and local blood vessel density. Assuming that the vascular density is taken to be $V=V_{M}$ within the viable tumour region, and $V=V_{N}$ elsewhere, we have

$\frac{\partial H}{\partial t}-D_{H} \nabla^{2} H= \begin{cases}0, & 0<R<R_{D}, \\ \phi_{Q} K_{M}-r_{V} V_{M} H, & R_{D}<R<R_{Q}, \\ \phi_{A} K_{M}-r_{V} V_{M} H, & R_{Q}<R<R_{M}, \\ -r_{V} V_{N} H, & R_{M}<R,\end{cases}$

where $K_{M}$ denotes the tumour cell density and $D_{H}$ the acid diffusion coefficient.

Following Greenspan (1972), we assume that the necrotic cellular debris continually disintegrates into simpler chemical compounds at a rate proportional to the core volume. These compounds flow into the surrounding tissue and the cell volume lost in this way is replaced by cells pushed inward through surface tension forces. Further assuming that the rate of cellular proliferation is constant per unit volume in the active proliferating region, we have

$\frac{\mathrm{d} R_{M}^{3}}{\mathrm{~d} t}=S\left(R_{M}^{3}-R_{Q}^{3}\right)-L R_{D}^{3}$.

Taking $\rho=\sqrt{r_{V} V_{N} / D_{H}}, H_{0}=\phi_{A} K_{M} / r_{V} V_{N}$ and $T=3 / S$, we may non-dimensionalize Eqs. (1) and (2) with $r=\rho R, h=H / H_{0}$ and $\tau=t / T$ to obtain

$\xi \frac{\partial h}{\partial \tau}-\left(\frac{\partial^{2} h}{\partial r^{2}}+\frac{2}{r} \frac{\partial h}{\partial r}\right)= \begin{cases}0, & 0<r<r_{D}, \\ \varepsilon-\psi^{2} h, & r_{D}<r<r_{Q}, \\ 1-\psi^{2} h, & r_{Q}<r<r_{M}, \\ -h, & r_{M}<r,\end{cases}$

$h\left(r_{i}\right)=h_{i}, \quad i=D, Q, N$

$r_{M}^{2} \frac{\mathrm{d} r_{M}}{\mathrm{~d} \tau}=r_{M}^{3}-r_{Q}^{3}-\gamma^{3} r_{D}^{3}$

where $\xi=S / 3 r_{V} V_{N}, \varepsilon=\phi_{Q} / \phi_{A} \ll 1, \psi=\sqrt{V_{M} / V_{N}}, r_{i}=\rho R_{i}, h_{i}=$ $H_{i} / H_{0}$ and $\gamma=\sqrt[3]{L / S}$. The data of Martin and Jain (1994) demonstrate a smooth $\mathrm{pH}$ gradient extending from the tumour edge into the peritumoural normal tissue. Hence we assume here that $\lim _{r \rightarrow \infty} h(r)=0$, i.e. that there is no excess acidity a long distance from the tumour, and further that $h$ and $h_{r}$ are continuous at internal boundaries $r=r_{D}, r_{Q}$ and $r_{M}$.

Typical parameter values are given in Table 1 . Given a tumour cell cycle length of 5 days we may estimate $S=1.6 \times 10^{-6} \mathrm{~s}^{-1}$, from which we find $\xi=2.24 \times 10^{-3} \approx 0$. This property that the acid diffusion timescale ( $\sim$ minutes) is much shorter than the

Table 1

Typical parameter values used in the model

\begin{tabular}{lll}
\hline Parameter & Value & Reference \\
\hline$\rho$ & $4.7 \mathrm{~cm}^{-1}$ & Martin and Jain (1994) \\
$H_{0}$ & $10^{-5} \mathrm{M}$ & Martin and Jain (1994) \\
$D_{H}$ & $1.08 \times 10^{-5} \mathrm{~cm}^{2} \mathrm{~s}^{-1}$ & Gatenby and Gawlinski (1996) \\
$\varepsilon$ & 0.01 & Patel et al. (2001) \\
$\gamma$ & 0.5 & Estimated \\
$H_{D}$ & $10^{-6} \mathrm{M}$ & Patel et al. (2001) \\
$H_{Q}$ & $10^{-6.4} \mathrm{M}$ & Patel et al. (2001) \\
$H_{N}$ & $10^{-6.8} \mathrm{M}$ & Patel et al. (2001) \\
$h_{D}$ & 0.1 & $=H_{D} / H_{0}$ \\
$h_{Q}$ & 0.04 & $=H_{Q} / H_{0}$ \\
$h_{N}$ & 0.01 & $=H_{N} / H_{0}$ \\
$\psi$ & $0-1$ & Estimated \\
$\sigma_{0}$ & 0.5 & Estimated \\
\hline
\end{tabular}


tumour growth timescale ( $\sim$ days) allows us to assume $\xi=0$-i.e. that the acid quickly redistributes and reaches equilibrium. Following Patel et al. (2001), we take $\varepsilon=0.01-$ i.e. the rate of acid production of quiescent tumour cells is two orders of magnitude less than their proliferating counterparts.

It can be argued that the various simplifying assumptions above are too unrealistic to represent complex biological phenomena such tumour growth. For example, whilst radial symmetry holds for early tumour growth, during later development tumours often become asymmetric; indeed the degree of irregularity of the tumour boundary may provide clinicians with useful prognostic information (Cross et al., 1994). Moreover, apoptosis and necrosis are dependent on a wide range of environmental parameters besides acidity, with subpopulations of a heterogeneous tumour responding differently to each cue. However, the aim of this paper is to highlight the role of acidity; under our assumptions the model remains analytically tractable and allows us to fully understand general tissue dynamics. These effects can subsequently be included in more complex models where analysis is not possible, such as Smallbone et al. (2007a).

\subsection{Avascular and vascular tumours, no quiescence}

In an earlier work (Smallbone et al., 2005), we investigated Eqs. (3)-(5) in the absence of quiescence (i.e. $h_{Q}=h_{D}$ ), but we reiterate the results here for completeness. Two cases were considered, both an avascular tumour $(\psi=0)$ and a vascular tumour whose blood vessel density was the same as that found in normal tissue $(\psi=1)$.

In the avascular case, a benign two-phase growth pattern is observed. Initially, the tumour grows exponentially, without a necrotic core. At a critical radius, a necrotic core begins to develop and the second phase of tumour growth begins. During this phase, we see very little change in tumour size. However, the necrotic core grows rapidly towards its equilibrium value. A similar growth pattern is observed in the vascular case when $h_{D}<1$, and is presented in Fig. 2; here we see formation of a necrotic core at radius $r_{M}=0.53\left(R_{M} \approx 1 \mathrm{~mm}\right)$ before the tumour reaches a final size of $r_{M}=0.8\left(R_{M} \approx 1.7 \mathrm{~mm}\right)$. If this critical parameter $h_{D}>1$, however, the vascular tumour will display invasive exponential growth, without the formation of a necrotic core.

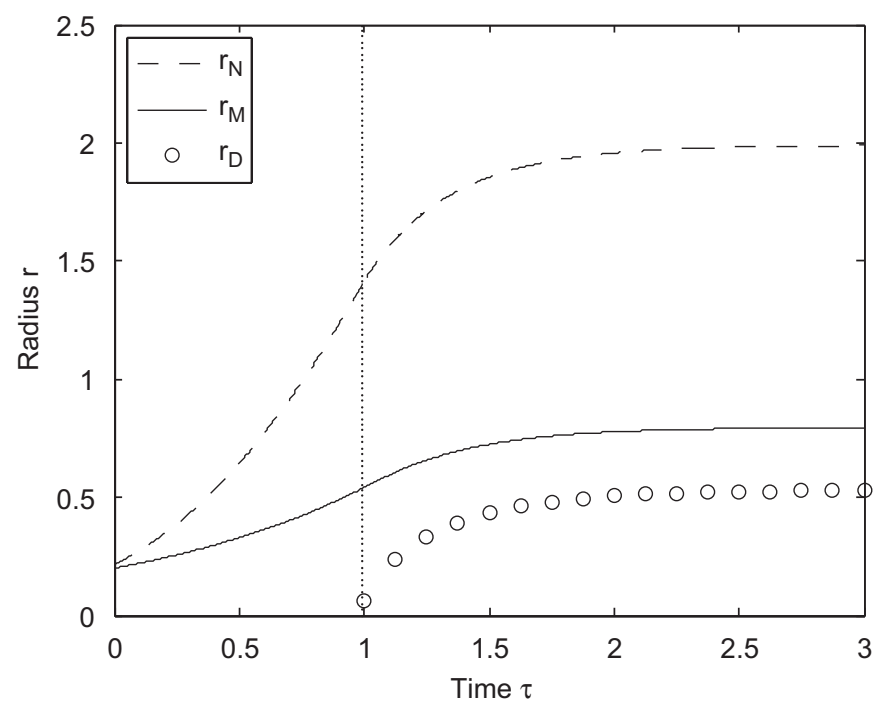

Fig. 2. Results from Eqs. (3) to (5). Recession of normal tissue accompanying vascular $(\psi=1)$ tumour growth, in the absence of quiescence $\left(h_{Q}=h_{D}\right)$. Parameter values used are $h_{N}=0.01, \gamma=0.5, h_{D}=0.1$ and $r_{M}(0)=0.2$.
Fig. 2 also shows normal tissue receding as the tumour grows. Notice the development of an acellular gap between the advancing tumour front and receding normal tissue, consistent with experimental observations (Gatenby and Gawlinski, 1996). However, one criticism that may be levelled at the model is the size of this acellular gap_predicted to be of a similar size to the tumour, and larger than the experimentally determined estimates of $100 \mu \mathrm{m}$.

\subsection{Avascular tumours with quiescence}

In this section, we extend the previous model through the inclusion of quiescent tumour cells, focussing first on avascular tumour growth (i.e. $\psi=0$ ), as this case is more amenable to analysis. Assuming as mentioned before that $\xi=0$, Eq. (3) has solution

$h(r)= \begin{cases}k_{1}, & 0<r<r_{D}, \\ k_{2}-k_{3} \frac{1}{r}-\varepsilon \frac{1}{6} r^{2}, & r_{D}<r<r_{Q}, \\ k_{4}-k_{5} \frac{1}{r}-\frac{1}{6} r^{2}, & r_{Q}<r<r_{M}, \\ k_{6} \frac{1}{r} \mathrm{e}^{-r}, & r_{M}<r,\end{cases}$

where the constants $k_{i}$ are given by

$k_{1}=\frac{2 \varepsilon r_{D}^{3}+2(1-\varepsilon) r_{Q}^{3}+r_{M}^{2}\left(r_{M}+3\right)}{6\left(r_{M}+1\right)}-\frac{\varepsilon r_{D}^{2}+(1-\varepsilon) r_{Q}^{2}}{2}$,

$k_{2}=k_{1}+\varepsilon \frac{r_{D}^{2}}{2}$

$k_{3}=\varepsilon \frac{r_{D}^{3}}{3}$

$k_{4}=k_{1}+\frac{\varepsilon r_{D}^{2}+(1-\varepsilon) r_{Q}^{2}}{2}$,

$k_{5}=\frac{\varepsilon r_{D}^{3}+(1-\varepsilon) r_{Q}^{3}}{3}$,

$k_{6}=\mathrm{e}^{r_{M}} \frac{-\varepsilon r_{D}^{3}-(1-\varepsilon) r_{Q}^{3}+r_{M}^{3}}{3\left(r_{M}+1\right)}$.

Assuming that acidity is the sole cause of necrosis and quiescence within the tumour allows us to calculate the radii of the necrotic core, $r_{D}$, and the quiescent region, $r_{Q}$, as functions of the tumour outer radius, $r_{M}$. While the tumour consists of only active proliferating cells (i.e. $r_{D}=r_{Q}=0$ ), from Eq. (6) we have

$h(0)=\frac{r_{M}^{2}\left(r_{M}+3\right)}{6\left(r_{M}+1\right)} \longrightarrow \infty \quad$ as $r_{M} \rightarrow \infty$.

Thus at some critical value $r_{M}=\hat{r}_{M}, h(0)=h_{Q}$, and the cells at the centre of the tumour will become quiescent. This critical radius is found by solving

$c_{1}\left(\hat{r}_{M}\right)=\hat{r}_{M}^{3}+3 \hat{r}_{M}^{2}-6 h_{Q} \hat{r}_{M}-6 h_{Q}=0$.

The positive solution $\hat{r}_{M}$ is given by Eq. (24), taking $n=0$.

If $r_{M}>\hat{r}_{M}$, then a quiescent region exists, and its radius $r_{Q}$ may be found by noting that the acid concentration at its boundary will be $h\left(r_{Q}\right)=h_{Q}$ :

$c_{2}\left(r_{Q}, r_{M}\right)=2(1-\varepsilon) r_{Q}^{3}-(3-2 \varepsilon)\left(r_{M}+1\right) r_{Q}^{2}+c_{1}\left(r_{M}\right)=0$.

The solution in $r_{Q}$ is given by Eq. (24), choosing $n=2$.

If $\varepsilon>0$, then we find that acidity will increase at the tumour boundary as it grows. Eventually all tumour cells will become quiescent, and the radius $\hat{r}_{Q}$ at which this occurs may be found through solution of Eq. (10), with $r_{Q}=r_{M}$ :

$\hat{r}_{Q}=\frac{3 h_{Q}+\sqrt{9 h_{Q}^{2}+12 h_{Q} \varepsilon}}{2 \varepsilon}$. 
Consider now the formation of necrosis within the tumour. While $r_{D}=0$ and $r_{Q}>0$, from Eq. (6) we have

$h(0)=h\left(r_{Q}\right)+\varepsilon \frac{r_{Q}^{2}}{6}=h_{Q}+\varepsilon \frac{r_{Q}^{2}}{6}$.

Thus setting

$h_{*}=h_{Q}+\varepsilon \frac{\hat{r}_{Q}^{2}}{6}=\frac{h_{Q}\left(\hat{r}_{Q}+3\right)}{2}$,

we see two distinct patterns of growth, dependent on the sign of $h_{D}-h_{*}$. If $h_{D}>h_{*}$, then no necrotic core will develop, and the tumour will grow to a state containing only quiescent cells. If $h_{D}<h_{*}$, however, at some critical value $r_{M}=\hat{r}_{D}, h(0)=h_{D}$ and the cells at the centre of the tumour will become necrotic. From Eq. (12), we find that this occurs when

$r_{Q}=r_{*}=\sqrt{\frac{6\left(h_{D}-h_{Q}\right)}{\varepsilon}}$.

$\hat{r}_{D}$ may then be found by solving $c_{2}\left(r_{*}, r_{M}\right)=0$ for $r_{M}$. This is achieved using Eq. (24), taking $n=0$.

If $r_{M}>\hat{r}_{D}$, then a necrotic core exists and its radius is given by noting that $h\left(r_{D}\right)=h_{D}$ :

$c_{3}\left(r_{D}, r_{Q}\right)=2 r_{D}^{3}-3 r_{Q} r_{D}^{2}+\left(r_{Q}^{2}-r_{*}^{2}\right) r_{Q}=0$.

The solution in $r_{D}$ is found using Eq. (24), taking $n=2$.

Furthermore, we know that $h\left(r_{Q}\right)=h_{Q}$ :

$c_{4}\left(r_{D}, r_{Q}, r_{M}\right)=r_{Q} c_{2}\left(r_{Q}, r_{M}\right)-2 \varepsilon\left(1+r_{M}-r_{Q}\right) r_{D}^{3}=0$.

Given $r_{M}$, we may numerically find $r_{Q} \in\left(r_{D}, r_{M}\right)$ from Eq. (16), using the expression for $r_{D}$ in Eq. (15).

The set of equations above allows calculation of the nondimensional quiescent tissue radius, $r_{Q}$, and the necrotic core radius, $r_{D}$, for any value of outer radius $r_{M}$. These radii may then be used to determine tumour growth from Eq. (5). Assuming that, at time 0 , the tumour is small enough that there is no necrotic core or quiescent region (i.e. $r_{D}=r_{Q}=0$ ), then the system is completely defined by Eqs. (5), (15) and (16), relying on parameters $\varepsilon, \gamma, h_{D}, h_{Q}$ and the initial condition $r_{M}(0)$.

Examples of the growth patterns observed are given in Fig. 3. Using typical parameter estimates of $h_{D}=0.1$ and $h_{Q}=0.04$, $h_{D}<h_{*}$ and so we see a three-phase growth. Initially, the tumour grows exponentially, whilst all cells are proliferative. At the critical radius $r_{M}=\hat{r}_{M}$, the central tissue becomes quiescent, restricting the active cells to a thin outer rim (Fig. 3(a)). At a later stage, when $r_{M}=\hat{r}_{D}$, we see the development of a necrotic core, followed by convergence of the tumour to its equilibrium size (Fig. 3(b)), behaviour typically seen in multicellular spheroids grown in vitro. In Fig. 3(c), we increase the tumour's susceptibility to acid-induced quiescence, taking $h_{Q}=0.01$, equivalent to $\mathrm{pH}$ 6.8. In this case, $h_{D}>h_{*}$, and no necrotic core will develop. Rather, it slowly grows to its equilibrium size $r_{M}=\hat{r}_{Q}$ where all the tumour cells are quiescent. This description of a quiescent tumour with no necrosis corresponds to a typical benign growth such as an adenoma.

We move on now to the acid-mediated invasion of normal tissue, and the corresponding development of an acellular gap separating the advancing tumour and receding host tissue fronts. The normal tissue front $r_{N}$ is defined by the relationship $h\left(r_{N}\right)=$ $h_{N}$ i.e. $k_{6} \mathrm{e}^{-r_{N}} / r_{N}=h_{N}$, which has solution

$r_{N}=W_{0}\left(\frac{k_{6}}{h_{N}}\right)$,

where $W_{0}$ denotes the principal value of the Lambert $W$ (or product $\log$ ) function-the inverse function of $f(W)=W \mathrm{e}^{W}$ (Corless et al., 1996). a

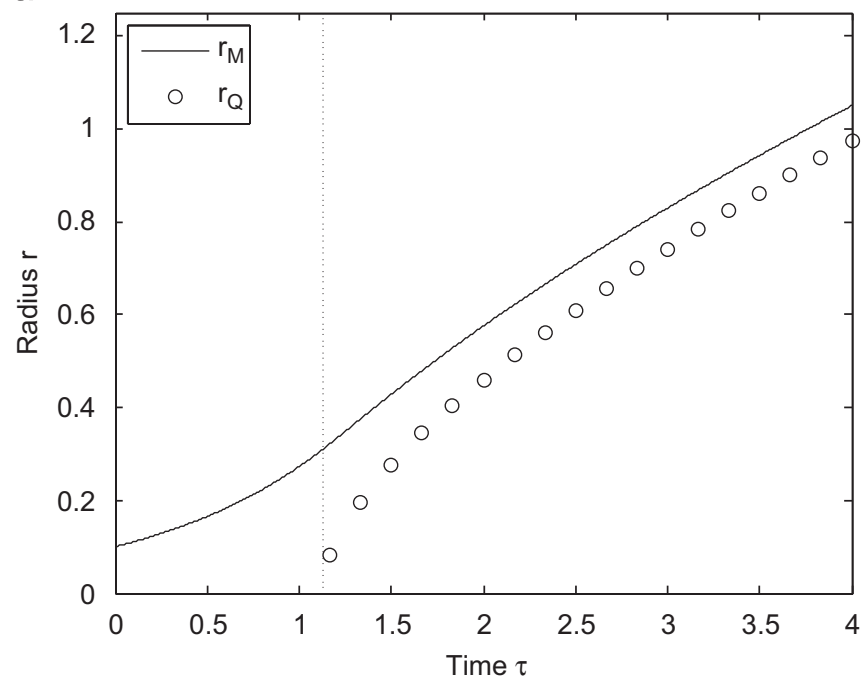

b

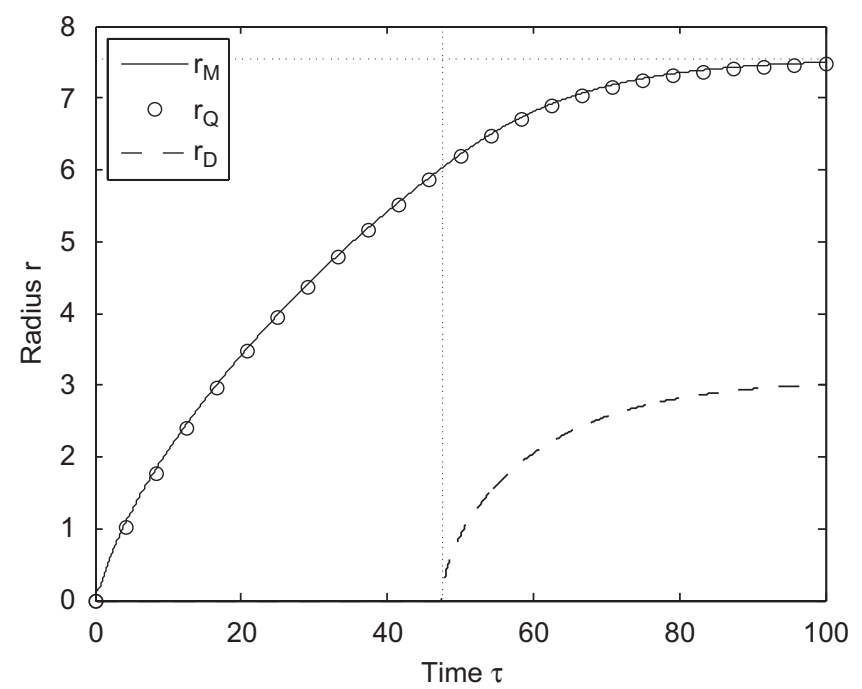

C

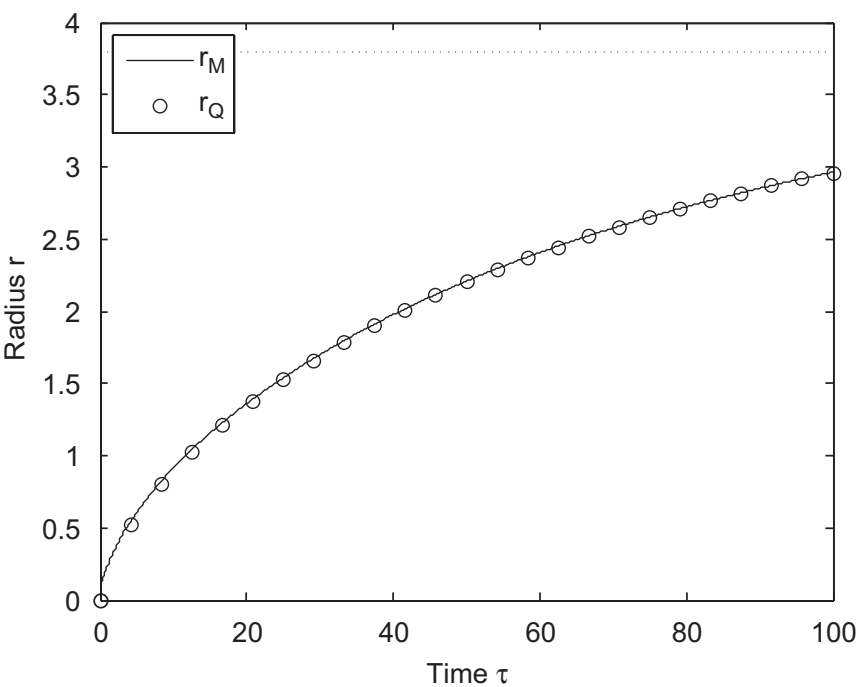

Fig. 3. Results from Eqs. (3) to (5). (a) Early stage and (b) late-stage avascular $(\psi=0)$ tumour growth with quiescence and necrosis, with parameters $\varepsilon=0.01$, $\gamma=0.5, h_{D}=0.1, h_{Q}=0.04$ and $r_{M}(0)=0.1$. (c) Non-necrotic growth with parameters $\varepsilon=0.01, h_{D}=0.1, h_{Q}=0.01$ and $r_{M}(0)=0.1$. 
In Fig. 3(b), we see that in necrotic growth, the layer of proliferating cells forms a very thin layer at the edge of the tumour, hence we may approximate $r_{M} \approx r_{Q}$ at equilibrium. In non-necrotic growth (c), there are no proliferating cells at equilibrium, hence $r_{M}=r_{Q}$. In both cases, we may assume that $h\left(r_{M}\right) \approx h\left(r_{Q}\right)=h_{Q}$. Then, from Eqs. (5) and (6) we find

$$
\begin{aligned}
\omega=r_{N}-r_{M} & \approx W_{0}\left(r_{M} \mathrm{e}^{r_{M}} \frac{h_{Q}}{h_{N}}\right) \\
& \approx\left(1+r_{M}^{-1}\right) \log \frac{h_{Q}}{h_{N}},
\end{aligned}
$$

where the second equation arises from taking the Taylor expansion about $r_{M}=\infty$.

In Fig. 4 we compare the interfacial width at equilibrium $\omega$ with changes in tumour quiescence threshold $h_{Q}$. We see that Eq. (19) represents a good approximation to the gap size $\omega$. Most importantly, through comparison of Figs. 3 and 4, we see that the equilibrium tumour width is predicted to be approximately 10 times as large as the interfacial width. This is significantly more physiologically accurate than the basic model (see Fig. 2), where the gap was predicted to be of a similar size to the tumour. Indeed, if we take the steady state tumour radius $r_{M}=\hat{r}_{Q}$ from Eq. (11), occurring in non-necrotic growth, and the approximate value for $\omega$ in Eq. (19), the gap to tumour ratio is given by

$\frac{w}{r_{M}}=\frac{\varepsilon}{3 h_{Q}} \log \frac{h_{Q}}{h_{N}}$

equal to 0.11 at our default parameter values.

\subsection{Vascular tumours with quiescence}

All of the analyses presented above are inherently reliant on the property that acidity levels increase as one moves toward the centre of the tumour. However, in the case of a vascularized tumour where we consider the effects of quiescence, this may not be the case, and the tumour may not adopt the typical geometry presented in Fig. 1.

Indeed in Smallbone et al. (2007b), we examined a simplified version of Eq. (3), ignoring the effects of necrosis (i.e. $r_{D}=0$, or equivalently $h_{D} \rightarrow \infty$ ). We found that the equation has no solution

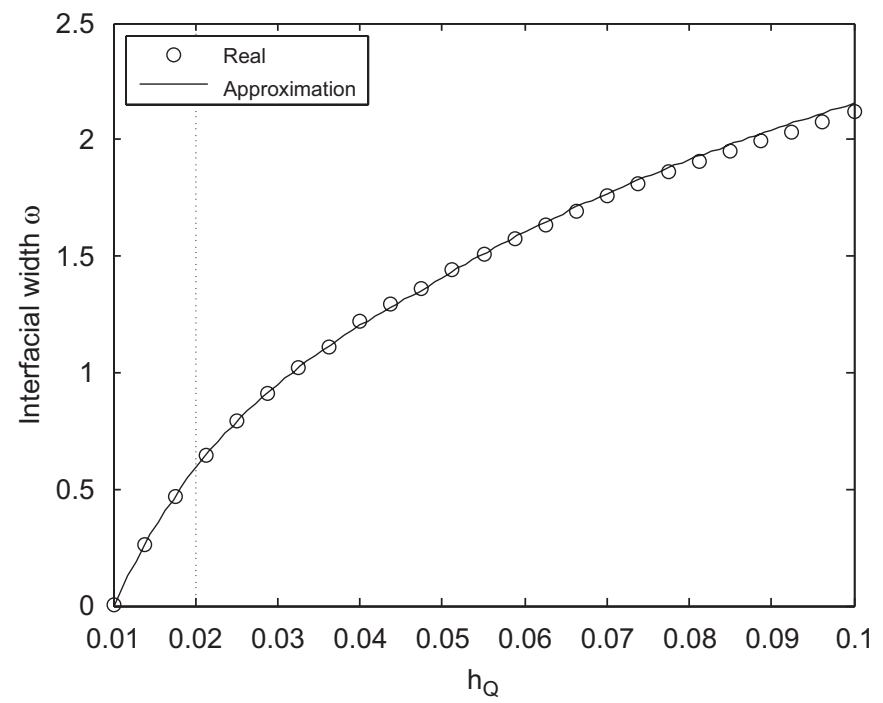

Fig. 4. Variation in size of acellular gap separating tumour and normal tissue fronts at equilibrium, with changes in the tumour quiescence threshold $h_{Q}$. The gap width is compared with the simple approximation in Eq. (19). Parameter values used are $\psi=0, \varepsilon=0.01, \gamma=0.5, h_{D}=0.1$ and $h_{N}=0.01$. The dotted line represents the crossover from non-necrotic to necrotic growth. $\xi=0$, provided $h_{Q} \psi^{2} \in(\varepsilon, 1)$ (as is typically the case), and the tumour is sufficiently large $\left(r_{M}>0.31\right.$, equivalent to $R_{M}>0.7 \mathrm{~mm}$ at our typical parameter values). This implies that the typical assumption of temporal homogeneity is not valid, and instead the full dynamics must be considered.

In the absence of necrosis, we may reformulate Eqs. (3) and (4) in terms of the Heaviside function $\theta$ :

$$
\begin{aligned}
\frac{\partial h}{\partial \sigma} & -\left(\frac{\partial^{2} h}{\partial r^{2}}+\frac{2}{r} \frac{\partial h}{\partial r}\right) \\
& = \begin{cases}(1-\varepsilon) \theta\left(h_{Q}-h\left(\sigma-\sigma_{0}\right)\right)+\varepsilon-\psi^{2} h, & 0<r<r_{M}, \\
-h, & r_{M}<r,\end{cases}
\end{aligned}
$$

where we change the timescale to that of acid diffusion $\sigma=\tau / \xi$ and introduce a lag term $\sigma_{0}$ between extracellular acid levels changing, and cells mounting the appropriate response of quiescence or proliferation.

A typical model solution is presented in Fig. 5. Taking $\sigma_{0}=0.5$, equivalent to a lag of $t_{0} \approx 30 \mathrm{~min}$, we investigate how acidity levels vary through the tumour. The model predicts that cycles of acidity will be seen: high levels of acidity induce cells to cease proliferation. Quiescent cells produce significantly less acid than their proliferating counterparts, thus the level of acidity will decrease, allowing cells to resume proliferation.

Initially, all the cells within the tumour are below the quiescence threshold. Cycles of acidity are out of phase for different sections of the tumour and an increase in acidity is first seen at the tumour edge $(r=1)$. Acidity then increases at the tumour centre $(r=0)$, before reaching its maximum level. This figure demonstrates that, whilst acid levels are on average higher in the tumour centre than the tumour edge, this property does not hold for all points in the acid cycle. Indeed the first cells to become quiescent due to extracellular acidity are not at the tumour centre; rather this occurs at $r \approx 0.81$ near the edge of the tumour.

Fluctuations in metabolite levels are known to occur within tumours with discrete periodicities of hours, minutes and days (Baudelet et al., 2004; Braun et al., 1999). Cells that are best suited to respond to these periods of cellular stress, such as through constitutive upregulation of aerobic glycolysis, will be positively

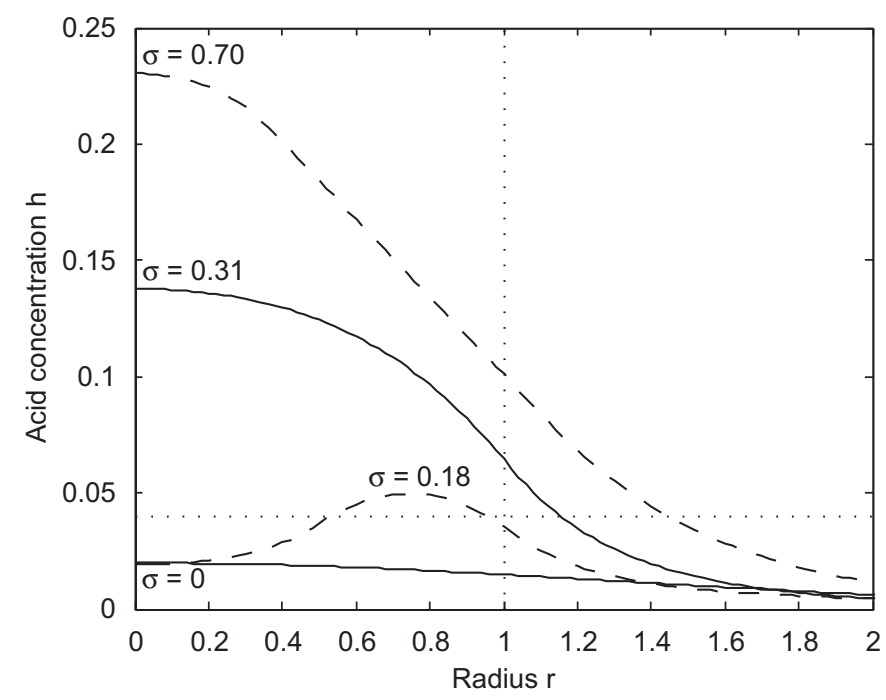

Fig. 5. Results from Eq. (21). Changing acid profiles in and around the tumour during an acidosis cycle. Notice that for some of this cycle, acidity is higher at the tumour edge than centre. Parameter values used are $h_{Q}=0.04, \varepsilon=0.01, \psi=1$, $\sigma_{0}=0.5$ and $r_{M}=1$. The dotted lines represent the tumour radius $\left(r_{M}=1\right)$ and quiescence threshold $\left(h_{Q}=0.04\right)$. The acidity levels cycle around the quiescence threshold, with cycle time of approximately 1.4 units. Adapted from Smallbone et al. (2007b). 
selected by somatic evolutionary forces. These cycles are assumed to occur due to hæmodynamic variations such as changes in the local concentration of red blood cells or structural rearrangement of blood vessels (Gatenby and Gillies, 2004). We have shown that an alternative hypothesis, namely quiescence in response to cellular stress and the corresponding drop in metabolism, provides a negative feedback mechanism capable of reproducing such metabolite cycles.

\section{Discussion}

In this paper, we have presented a selection of models of both vascular and avascular tumour growth, where the invasion mechanism is the acidification of the microenvironment surrounding the tumour due to increased reliance on glycolysis. A major criticism of an earlier model was that the width of the interfacial acellular gap was predicted to be of a similar size to the tumour, larger than has been observed experimentally. Within avascular tumours in particular, the vast majority of the viable cells are quiescent, producing significantly less acid than their proliferating counterparts. As such, we extend the basic model to include the effects of quiescent, as well as proliferating and necrotic tumour cells.

Analysis of the avascular model predicts two regimes of tumour growth, dependent on a relationship between the thresholds for acidity-induced tumour cell quiescence $h_{Q}$ and tumour cell death $h_{D}$. If the tumour is sufficiently resistant to acid-induced cell death, we predict that no necrotic core will develop, and the tumour will grow to a state containing only quiescent tissue. Conversely, if the resistance $h_{D}$ drops below a critical threshold $h_{*}$, we predict that, at equilibrium, the proliferative tumour tissue will be limited to a thin, relatively alkaline, region at the tumour edge, whilst the core of the tumour will contain necrotic material. At steady state, proliferation at the rim is counterbalanced by volume loss through degradation of the necrotic core.

In both cases, we predict that the advancing tumour front is separated from the receding normal tissue by an acellular gap. In contrast to earlier work, we find that the gap width is an order of magnitude smaller than the tumour radius at equilibrium. A simple functional approximation (Eq. (19)) allows us to calculate the expected width of this gap in terms of the tumour radius, the tumour tissue quiescence threshold $\left(h_{Q}\right)$ and the host tissue death threshold $\left(h_{N}\right)$.

As blood vessels are the primary mode for waste removal, within avascular tumours we see a build-up of acid, limiting growth through auto-toxicity. To demonstrate sustained growth, tumours must acquire a vascular bed; if the vascular density rises sufficiently, the tumour avoids excess acid accumulation and may invade the whole of the normal tissue space. However, the introduction of quiescence into the model adds an interesting new dynamic: cycles of acidity may be observed in the tumour. Previously, variations in substrate levels were assumed to occur through oscillations in the hæmodynamics of nearby and distant blood vessels. Within our modelling framework, quiescent cells produce less acid than their proliferating counterparts. Through vascular removal, acidity levels decrease, in time allowing cells to resume proliferation. This simple negative feedback mechanism is shown to produce the observed cycles in tumour substrate levels.

Simple reaction-diffusion systems are used here to describe the dynamics of acidity in and around a tumour. Tumour growth is dependent on the complex interactive dynamics of many different factors, including the supply of nutrients and growth factors and the specific mutations displayed by the tumour population. Moreover, inhomogeneities can play an important role, and stochastic or cellular automaton models (Patel et al., 2001; Smallbone et al., 2007a) may be more appropriate than the mean-field approach used here. However, using simplifying assumptions we have shown that increased tumour acid production alone, almost universally observed in clinical cancers, is sufficient to explain a variety of behaviours; these include benign and invasive development, an interstitial gap separating the tumour and host tissue fronts, and cycles of acid levels driven by cellular quiescence. Moreover, the tractability of the model has allowed us to identify the key parameters controlling the change between different growth regimes.

\section{Acknowledgements}

KS acknowledges the support of the BBSRC/EPSRC Grant BB/ C008219/1 "The Manchester Centre for Integrative Systems Biology (MCISB)". PKM was partially supported by a Royal Society Wolfson Merit Award.

\section{Appendix}

Given a cubic equation

$f(x)=x^{3}+a_{2} x^{2}+a_{1} x+a_{0}=0$,

define

$P=\frac{3 a_{1}-a_{2}^{2}}{9}$

$Q=\frac{9 a_{1} a_{2}-27 a_{0}-2 a_{2}^{3}}{54}$.

Then, if the polynomial discriminant $D=P^{3}+Q^{2}<0$, all solutions to Eq. (22) are real and unequal, and are given by

$x_{n}=2 \sqrt{-P} \cos \left(\frac{\theta+2 n \pi}{3}\right)-\frac{a_{2}}{3}$,

where

$\theta=\arccos \left(\frac{Q}{\sqrt{-P^{3}}}\right)$

for $n=0,1,2$, where we choose arccos : $[-1,1] \rightarrow[0, \pi]$.

\section{References}

Baudelet, C., Ansiaux, R., Jordan, B.F., Havaux, X., Macq, B., Gallez, B., 2004. Physiological noise in murine solid tumours using $\mathrm{T} 2{ }^{\star}$-weighted gradientecho imaging: a marker of tumour acute hypoxia? Phys. Med. Biol. 49, 3389-3411.

Braun, R.D., Lanzen, J.L., Dewhirst, M.W., 1999. Fourier analysis of fluctuations of oxygen tension and blood flow in R3230Ac tumors and muscle in rats. Am. J. Physiol. 277, H551-H568.

Casciari, J.J., Sotirchos, S.V., Sutherland, R.M., 1992. Variations in tumor cell growth rates and metabolism with oxygen concentration, glucose concentration, and extracellular pH. J. Cell. Physiol. 151, 386-394.

Corless, R.M., Gonnet, G.H., Hare, D.E.G., Jeffrey, D.J., Knuth, D.E., 1996. On the Lambert W function. Adv. Comput. Math. 5, 329-359.

Cross, S.S., Bury, J.P., Silcocks, P.B., Stephenson, T.J., Cotton, D.W.K., 1994. Fractal geometric analysis of colorectal polyps. J. Pathol. 172, 317-323.

Gatenby, R.A., Gawlinski, E.T., 1996. A reaction-diffusion model of cancer invasion. Cancer Res. 56, 5745-5753.

Gatenby, R.A., Gillies, R.J., 2004. Why do cancers have high aerobic glycolysis? Nat. Rev. Cancer 4, 891-899.

Gillies, R.J., Raghunand, N., Karczmar, G.S., Bhujwalla, Z.M., 2002. MRI of the tumor microenvironment. J. Magn. Reson. Imaging 16, 430-450.

Goda, N., Ryan, H.E., Khadivi, B., McNulty, W.C., Rickert, R.C., Johnson, R.S., 2003. Hypoxia-inducible factor $1 \alpha$ is essential for cell cycle arrest during hypoxia. Mol. Cell. Biol. 23, 359-369.

Greenspan, H.P., 1972. Models for the growth of a solid tumor by diffusion. Stud. Appl. Math. 51, 317-340. 
Martin, G.R., Jain, R.K., 1994. Noninvasive measurement of interstitial pH profiles in normal and neoplastic tissue using fluorescence ratio imaging microscopy. Cancer Res. 54, 5670-5674.

Murphy, M., Carlson, J.A., Keough, M.P., Claffey, K.P., Signoretti, S., Loda, M., 2004. Hypoxia regulation of the cell cycle in malignant melanoma: putative role for the cyclin-dependent kinase p2 $7^{\text {kip } 1}$. J. Cutan. Pathol. 31, 477-482.

Patel, A.A., Gawlinski, E.T., Lemieux, S.K., Gatenby, R.A., 2001. A cellular automaton model of early tumor growth and invasion. J. Theor. Biol. 213, 315-331.
Smallbone, K., Gavaghan, D.J., Gatenby, R.A., Maini, P.K., 2005. The role of acidity in solid tumour growth and invasion. J. Theor. Biol. 235, 476-484.

Smallbone, K., Gatenby, R.A., Gillies, R.J., Maini, P.K., Gavaghan, D.J., 2007a. Metabolic changes during carcinogenesis: potential impact on invasiveness. J. Theor. Biol. 244, 703-713.

Smallbone, K., Gavaghan, D.J., Maini, P.K., Brady, J.M., 2007b. Quiescence as a mechanism for cyclical hypoxia and acidosis. J. Math. Biol. 55, 767-779.

Thomlinson, R.H., Gray, L.H., 1955. The histological structure of some human lung cancers and the possible implications for radiotherapy. Br. J. Cancer 9, 539-549.

Warburg, O., 1930. The Metabolism of Tumours. Constable Press, London. 